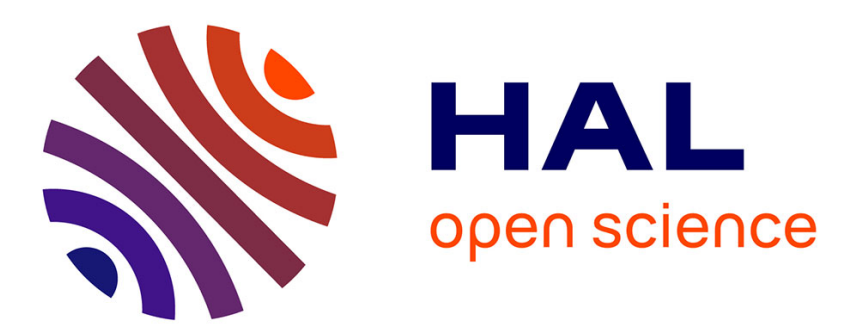

\title{
Les objets intermédiaires de conception / design, instruments d'une recherche par le design
}

Jean-François Bassereau, Régine Charvet Pello, Jenny Faucheu, David

Delafosse Delafosse

\section{- To cite this version:}

Jean-François Bassereau, Régine Charvet Pello, Jenny Faucheu, David Delafosse Delafosse. Les objets intermédiaires de conception / design, instruments d'une recherche par le design. Sciences du Design, 2015, n 2 (2), pp.48. 10.3917/sdd.002.0048 . emse-02916244

\section{HAL Id: emse-02916244 \\ https://hal-emse.ccsd.cnrs.fr/emse-02916244}

Submitted on 17 Aug 2020

HAL is a multi-disciplinary open access archive for the deposit and dissemination of scientific research documents, whether they are published or not. The documents may come from teaching and research institutions in France or abroad, or from public or private research centers.
L'archive ouverte pluridisciplinaire HAL, est destinée au dépôt et à la diffusion de documents scientifiques de niveau recherche, publiés ou non, émanant des établissements d'enseignement et de recherche français ou étrangers, des laboratoires publics ou privés. 


\title{
LES OBJETS INTERMEDIAIRES DE CONCEPTION/DESIGN, INSTRUMENT D'UNE RECHERCHE PAR LE DESIGN
}

\author{
Jean-François Bassereau, Régine Charvet Pello, Jenny Faucheu, David Delafosse
}

\section{Résumé}

Le design se pratique en projet de conception d'objets. Le plus souvent, ce n'est pas le seul métier ou discipline à intervenir. Dans tout projet de conception/design, l'utilisation et la manipulation d'information sont vitales. Le support de l'information doit accompagner le dialogue entre les métiers. Si le dessin technique permet l'échange entre les métiers de la conception et ceux de la réalisation, il nécessite un apprentissage. Le dessin technique est un objet intermédiaire de conception codifié, il suppose d'être appris pour être compris. Le design possède de nombreux supports d'information propice à l'échange « naturelle » entre les métiers (croquis, dessin, rough, maquette d'étude, maquette d'aspect). Pourtant, dans ces cas, a contrario du dessin technique, un certain flou d'interprétation peut exister favorisant des quiproquos.

Expliciter les outils de (re)présentation du design devient un sujet d'une recherche interdisciplinaire par le design. Le design sensoriel a hybridé les outils de l'évaluation sensorielle en les orientant projet de conception. Quelques outils méthodologiques illustrent ce que peut être une recherche par le design.

MOTS-CLÉS : Outil méthodologique, design sensoriel, Objet Intermédiaire de conception, recherche design

\begin{abstract}
Design practise takes the form of a project. Most often, it is not the only profession or discipline to intervene. In any design project, use and handling of information are crucial. The support of information must accompany the dialogue between trades. If technical drawing allows the exchange between the domains of design and those of engineering, it requires a learning. The technical drawing is an intermediate object of codified design, it is assumed to be learned to be understood. Design produces many information tools for the natural exchanges between trades (sketches, drawings, roughs, study models, appearance models). But, contrary to the technical drawings, some uncertainty of interpretation may exist and foster misunderstandings.

Explaining the design representation tools becomes a subject of interdisciplinary research by design. The sensory design has hybridized the sensory evaluation tools by orienting them in the design project. Some methodological tools illustrate what kind of research can be achieve by design.
\end{abstract}

Key Words : Methodological tool, sensory design, Intermediary Objects of design, design research

\section{Introduction}

La question récurrente commune à la conception de produits et la création d'objets concerne la transmission et la traduction matérielle d'une idée. Problème d'autant plus crucial quand une idée individuelle doit se partager à plusieurs, dans et par des métiers différents. En outre, le contexte de l'ingénierie simultanée et des plateaux projets encouragent la rencontre des différents acteurs de la conception qui ne parlent pourtant jamais la même langue, pire emploient des mots similaires aux significations différentes suivant les métiers et disciplines. C'est un des premiers paradoxes de 
l'ingénierie simultanée, le paradoxe de Babel $^{1}$, né d'une réponse partielle à un problème complexe. II provient du projet de conception/design qui, même simples, se doivent d'être étudiés dans leurs attributs divers par une approche interdisciplinaire. Or, il ne suffit pas de recenser les disciplines et métiers nécessaires à la conception et les mettre au travail. L'échange d'information entre métiers différents ne va pas de soi et peut poser des problèmes à tiroirs (Minel, 2003 ; Simon, 2006).

Si le projet de conception de tout nouvel objet nécessite bien une pratique interdisciplinaire, liée à la conception/design de tous les attributs du nouvel objet et de ces conséquences des plus directes au plus indirectes, ces exigences obligent de dépasser le paradoxe de Babel. La première tentative de dépassement mobilise un concept méthodologique des praticiens/chercheurs en conception de produits enrichi par un regard de sociologues (Tichkievitch et Mer, 1995 ; Jeantet, 1998 ; Boujut, 2001) ; l'Objet Intermédiaire de conception (aussi appelé « (re)présentation intermédiaire ", (simplifié dans l'abréviation OI)².

\section{Les objets Intermédiaires de conception, premières conceptualisations}

Dans l'activité de design, la génération de représentations intermédiaires est courante, tels que dessin, maquette, croquis, rough, croquis coté, démonstrateur, (planche et panneau de tendance parfois) et prototype ${ }^{3}$. Elles participent depuis l'origine à la formation initiale du designer, jalonnent parfois les avancées de ses prestations et servent de soutien à sa pensée, son projet. Peu de praticiens ont éprouvé le besoin d'expliciter le principe constructif qui les génère (Pesce, 1996 ; Caraes et al., 2011). Les représentations intermédiaires de conception apparaissent dès que la pratique de la conception a nécessité une conceptualisation des décisions intuitives sans éléments prédictifs disponibles. A l'origine, pour aider le concepteur dans ses activités solitaires, dans une mise en dialogue avec lui-même nécessaire, manipulant à travers un média représentationnel ses propres représentations intellectuelles. Puis, dès que la pratique de la conception/réalisation a rassemblé plus d'un acteur, la représentation intermédiaire s'est imposée comme une garantie, un langage facilitant un dialogue possible, avec certaines orientations et prises de décision partagées.

Le partage et la transmission des connaissances (génériques, métiers et spécifiques au projet), plus largement de toutes données entre concepteur et réalisateur, ont vu la nécessité d'une codification de la représentation graphique, pour cadrer les interprétations, la rendant en conséquence moins immédiate et naturelle dans sa lecture. Les maquettes apparaissent comme plus lisibles, sans apprentissage particulier pour les comprendre. Elles appellent souvent des techniques non triviales et constituent une catégorie d'objets intermédiaires de conception où la lecture peut être parfois faussement intuitive.

\footnotetext{
${ }^{1}$ Le paradoxe de Babel (Bassereau, 2013) représente un risque de blocage du projet comme celui de la construction de la tour dont les Dieux sont responsables. Inquiets d'être dérangés par cette construction ils ont doté à chaque groupe de travailleurs une langue différente des autres. Ne se comprenant plus la construction de la tour de Babel a cessé. Or, construire une tour aussi haute ne peut se faire seul.

${ }^{2}$ L'objet final obtenu au terme du projet, constitue une catégorie particulière d'O I, à un niveau macro et micro. Au niveau microscopique, entre le concepteur et l'utilisateur, et au niveau macroscopique, à l'échelle de la conception (son histoire). Leroi Gourhan l'illustre avec l'exemple du sabre, dans son ouvrage milieu et techniques en 1945. Il nous explique que l'atteinte de l'objet idéal, comme le sabre idéal a pris des millénaires, émaillée d'essais/erreurs. Seuls les sabres qui ont démontré leur utilité ont survécu et perduré. Les sabres essais et les sabres erreurs ont constitué des représentations intermédiaires sous forme d'objets qui s'étendent sur des décennies, à ramener dans une temporalité de projets de conception. Lorsque la réalisation de l'objet définitif fut trop longue, impliquant, onéreuse des représentations intermédiaires furent mobilisées.

${ }^{3}$ Elles constituent une catégorie à part dans la typologie des objets intermédiaires tels que définis par des chercheurs sur la pratique de la conception, comme des chercheurs praticiens tant en conception qu'en design, Boujut . « Nous désignons comme représentations intermédiaires, appelées aussi objets intermédiaires ("Intermediary objects of design (IODs) " [Papadimitriou et al. 96], "Objets intermédiaires » [Mer et al. 95] [Vinck 09]) ou objets-frontières (" Boundary objects " [Carlile 02] [Carlile 04] [Broberg 10]) dans les études scientifiques, tous les types d'objets qu'ils soient physiques (plans, maquettes, croquis etc.) ou virtuels (CAO, résultats de calculs, etc.) produits par les participants au cours de leurs travaux [de conception]. En bref, cela comprend tous les types d'externalisation » Traduit de la définition en anglais : " Intermediary object is a general category embracing all types of artefacts, whether physical (plans, mock-ups, sketches, etc.) or virtual (CAD models, calculation results, etc.), produced by the participants during their work. In other words, it covers all kinds of externalisation. " (p. 509) [Boujut et al. 03].
} 
Qu'elles soient tangibles ou virtuelles, toutes les représentations intermédiaires ont deux fonctions indissociables, comme le rappelle Mer (1995), à savoir : modéliser la réalité et permettre la coordination et la coopération entre les acteurs du projet. Leurs diversités formelles appellent à une clarification sous une forme opératoire, exigée par le cadre conceptuel de l'action recherche ${ }^{4}$. Plusieurs chercheurs en génie industriel, mécanique se sont intéressés à l'étude des représentations intermédiaires. Ainsi, Carlile, cité par Costes en $2012^{5}$ a déterminé trois catégories de représentations intermédiaires au cours du processus de conception (Cf. Tableau $\mathrm{N}^{\circ} 1$ ).

\begin{tabular}{|c|l|l|}
\hline CATEGORIES & \multicolumn{1}{|c|}{ DESIGNATIONS } & \multicolumn{1}{|c|}{ EXEMPLES } \\
\hline Catégorie 1 & repertories & $\begin{array}{l}\text { - cost databases } \\
\text { - CAD / CAM databases } \\
\text { - parts librairies }\end{array}$ \\
\hline Catégorie 2 & standardized forms and methods & $\begin{array}{l}\text { - standars for reporting findings } \\
\text { - problem solving methods } \\
\text { - engineering change forms }\end{array}$ \\
\hline Catégorie 3 & objects, models and maps & $\begin{array}{l}\text { - sketches, assembly drawings, parts } \\
\text { - prototype assemblies } \\
\text { - mock-ups, computer simulation, Gantt charts } \\
\text { - process maps }\end{array}$ \\
\hline
\end{tabular}

Tableau N 1 - Catégorisation des représentations intermédiaires d'après [Carlile 02 ; 04]

Ce premier classement a le mérite d'identifier une typologie de catégories, mais celui de Mer, dès 1995, est à la fois plus complet et plus appropriable. II propose d'étudier les représentations intermédiaires selon deux axes complémentaires liés au dialogue collaboratif lors d'un projet de conception/design :

$1^{\circ}$ ) L'axe lié à la communication entre concepteurs et utilisateurs place l'O. I., d'un côté en porteur de message direct et de l'autre en messager intermédiaire.

Objet commissionnaire <-------------> objet médiateur ${ }^{6}$

\footnotetext{
4 «L'Action Recherche est la dernière évolution conceptuelle que je propose comme cadre de référence théorique de la conception, du design abritant en son sein des activités potentielles tout à la fois liées au projet de conception d'un objet (et non plus d'un produit) et liées à la constitution d'une connaissance. A propos de la constitution de celle-ci, il est clair que dès la naissance du design (mais bien avant, aux prémices de la conception, (Cf. histoire raisonnée de la conception), les actions et les démarches exploratoires, voir introspectives, ont été liées et mises au service d'un projet, source de connaissances multiples, chassant, du même coup, toute coupure entre activités manuelles et intellectuelles. En effet, en conception/création d'objets et dans toutes les connaissances sollicitées pour y parvenir cette distinction n'a pas de justification ». « S'il fallait résumer quelques traits caractéristiques complémentaires de la forme de recherche en design, nous dirions, en paraphrasant une expression connue, qu'elle suscite un dire sur le faire capable d'éclairer le faire et, indissociablement, un faire irrigué par un dire dont l'ancrage dans le faire garantit la fécondité ». FINDELI A. (2008), Introduction aux Ateliers de la Recherche en Design, Nîmes, juin. Cette indissociation des modalités du faire, ancrage possible d'une forme de connaissances incorporées (au sens de construit, contenu et ancré dans le corps), renvoie aux origines de l'enseignement du design au Bauhaus. Maîtres d'Atelier et enseignants formaient un Janus pédagogique qui concrétisait le concept de " main qui pense ». Récursivité des causes et des effets qui rompt avec le modèle classique des sciences. Ici, en recherche en et par le design, ce n'est pas tant le projet de recherche qui enclenche les actions, mais le projet design qui invite à la recherche par une démarche exploratoire souvent expansive, même si elle reste orientée par le projet design. Dans ce cas, et dans ce cas seulement, il faut plus précisément parler, pour situer la nature d'une recherche en design, d'action recherche, plutôt que de recherche action, historiquement liée aux sciences sociales modernes. Cette indissociabilité de la recherche et de l'action conduit à une pensée et une modalité du faire dialectique. «Pensée dialectique et complexe, assurément, que représente assez fidèlement un ruban de Möbius sur lequel théorie et pratique sont ni dedans ni dehors, ni avant ni après, ni causante ni causée, mais toujours néanmoins l'un et l'autre ». (FINDELI, 2008)

${ }^{5}$ La classification en trois catégories de Carlile a le mérite d'être simple, elle est directement relative à l'organisation du projet de conception. En effet, les représentations intermédiaires sont uniquement classées par fonction, du point de vue du concepteur qui les utilise pour concevoir un produit.

${ }^{6} \mathrm{Il}$ est dit médiateur car il prend place entre l'idée du concepteur (sa représentation mentale) et l'usage fait par le récepteur de la représentation intermédiaire. Mer choisit de décrire les interactions qui existent entre le produit et ses utilisateurs pour décrire cet axe. Ainsi «l'acteur utilisant un objet commissionnaire est en interaction, à travers l'objet, avec les intentions, les idées du producteur de l'objet même si elles sont quelque peu déformées. En revanche l'utilisateur d'un objet médiateur est en
} 
L'objet commissionnaire transmet l'idée, l'intention fidèle de son auteur alors que l'objet médiateur modifie l'intention initiale de son auteur du fait de sa matérialisation.

$2^{\circ}$ ) Le deuxième axe est tourné vers l'appropriation. Il ouvre deux possibilités antinomiques; soit l'O. I. accepte des modifications éventuelles dictées par une adaptation nécessaire au contexte du projet, il est dit « ouvert ». Soit l'O. I. ne supporte aucune modification possible sous peine de bug irrémédiable ; l’O. I. est dit « fermé ».

$$
\text { Objet ouvert <-------------> objet fermé }
$$

L'objet ouvert est une représentation intermédiaire qui peut être modifiée. A l'inverse, l'objet fermé ne permet et ne supporte aucune modification. Selon les travaux de Mer et al. en 1995 : " La notion d'ouverture est liée à un objet laissant à l'utilisateur une marge de manœuvre au sein de laquelle il peut plus ou moins diverger. En revanche, un objet fermé diminue et tend à faire disparaître cette marge de manœuvre. L'objet ouvert incite à un travail d'interprétation, tandis que l'objet fermé transmet une prescription».

Nous postulons sur le fait que le deuxième axe constitué doit rester complémentaire au premier et devra s'y combiner. Costes (2012) note que ces deux axes complémentaires décrivent les fonctions de représentations du point de vue du concepteur. Sur ce point, il rejoint donc Carlile $((2002,2004)$. Toutefois, Mer apporte une dimension supplémentaire en mettant en perspective les représentations intermédiaires par rapport à ses utilisateurs. Pour poursuivre cet effort de précision, nous précisons une typologie des modes de représentations complétée par le nombre de dimensions représentées, présentes tout au long du projet de conception/design, dans un objectif double d'explicitation et d’opérabilité (Cf. Tableau $\mathrm{N}^{\circ} 2$ ).

\begin{tabular}{|l|l|l|}
\hline $\begin{array}{l}\text { NOMBRE DE } \\
\text { DIMENSIONS }\end{array}$ & MODE DE REPRESENTATION & ESPACE DE REPRESENTATION \\
\hline 3D & $\begin{array}{l}\text { Maquette } \\
\text { Prototype }\end{array}$ & Espace produit \\
\hline 2D & $\begin{array}{l}\text { Dessins } \\
\text { Croquis, nomenclature } \\
\text { Roughs, perspective (3D représenté), dessin technique } \\
\text { Descriptif, Cahier des charges, Interview, brief... }\end{array}$ & $\begin{array}{l}\text { Espace graphique } \\
\text { Espace sensorielle } \\
\text { Espace sémantique } \\
\text { Espace linguistique }\end{array}$ \\
\hline
\end{tabular}

Tableau $N^{\circ} 2$ - Typologie des modes de représentation

Au sein de ces deux catégories (2D /3D), les modes de représentation sont indiquées en fonction de leurs niveaux de représentation, autrement dit en fonction de leurs niveaux de précision (variable au cours du projet de conception/design). Chaque mode de représentation se réfère à un espace de représentation particulier, c'est-à-dire à un niveau cognitivo-perceptif de référence différent potentiellement les uns des autres.

Souvent, le projet de conception/design se représente sous forme de processus. L'O. I. se place en alternative à cette représentation. II se précise utilement et plus spécifiquement pour la catégorie des maquettes, plus largement pour toute représentation intermédiaire manipulant les trois dimensions. Ainsi, la notion de maquette " bonne sensation » apparaît comme un apport d'explicitations en et par

interaction avec l'objet lui-même. Dans cette situation, l'objet devient «acteur ». Il médiatise, au moins partiellement, le processus de conception antérieur. Représentant une partie de la conception, il fonctionne cependant "par lui-même » et agit comme un acteur à part entière. ». MER S. JEANTET A. TICHKIEWITCH S. (1995) - « Les objets intermédiaires de la conception : modélisation et communication », Le communicationnel pour concevoir, Europia, Ed. Caelen Jean et Zreik Khaldoun. p. 30. 
le design sensoriel. Elle illustre un effort de clarification qui vise à supprimer le flou possible ${ }^{7}$ entre maquette du design, comme système de conception auto centrée, destinée à la matérialisation d'une idée conceptuelle à travailler en itération avec la représentation graphique pour le designer lui-même, et une maquette du design comme mode de (re)présentation, visant à présenter soit à un client, soit aux autres acteurs du projet, le résultat intermédiaire de son travail. En spécifiant la nature " sensorielle » de la maquette, il devient alors possible de préciser son effort de conception/design et cadrer sa lecture (discussion/validation), au même niveau potentiel d'information que les autres $\mathrm{O}$. I. de conception, mais provenant d'autres métiers.

\section{L'exemple d'un O. I. du design sensoriel ; la maquette « bonne sensation »}

Parmi les représentations intermédiaires en 3D, seuls les attributs techniques et fonctionnels de l'objet sont représentés dans les objets intermédiaires étudiés jusqu'ici. Aucun espace de (re)présentation sensorielle n'est présent consciemment (en dehors de la modalité sensorielle visuelle, installée depuis longtemps en monopole implicite de tout système de représentation et de fonctionnement du projet) et mobilisé explicitement au sein du projet de conception (Cf. Tableau $\mathrm{N}^{\circ} 3$ ).

\begin{tabular}{|c|c|c|c|}
\hline & \multicolumn{3}{|l|}{ EVALUATION EN INTERNE } \\
\hline & $\begin{array}{l}\text { MAQUETTE } \\
\text { BONNE SENSATION }\end{array}$ & PROPRIÉTÉS & $\begin{array}{l}\text { FONCTION AU COURS DU } \\
\text { PROCESSUS DE CONCEPTION }\end{array}$ \\
\hline \multirow[t]{6}{*}{$3 \mathrm{D}$} & Maquette " bon toucher " & $\begin{array}{l}\text { Bonnes sensations } \\
\text { somesthésiques }\end{array}$ & $\begin{array}{l}\text { Validation de la matière, sa mise } \\
\text { en forme et ses états de surface }\end{array}$ \\
\hline & $\begin{array}{l}\text { Maquette « bonne } \\
\text { sonorité » }\end{array}$ & Bonnes sensations sonores & $\begin{array}{l}\text { Validation de la matière, sa mise } \\
\text { en forme et ses états de surface }\end{array}$ \\
\hline & Maquette « bonne densité » & $\begin{array}{l}\text { Bon rapport poids, volume } \\
\text { perçu }\end{array}$ & $\begin{array}{l}\text { Validation de la matière, sa mise } \\
\text { en forme }\end{array}$ \\
\hline & $\begin{array}{l}\text { Maquette " bonne } \\
\text { répartition des masses» }\end{array}$ & $\begin{array}{l}\text { Lors de la prise en main, } \\
\text { bonne répartition des } \\
\text { composants }\end{array}$ & $\begin{array}{l}\text { Validation de la matière, sa mise } \\
\text { en forme et son architecture }\end{array}$ \\
\hline & $\begin{array}{l}\text { Maquette « bonne prise en } \\
\text { main », « bons gestes » }\end{array}$ & $\begin{array}{l}\text { Interface individu maquette } \\
\text { validée }\end{array}$ & $\begin{array}{l}\text { Validation de la matière, sa mise } \\
\text { en forme }\end{array}$ \\
\hline & $\begin{array}{l}\text { Maquette « bonne } \\
\text { brillance ", etc. } \\
\text { Maquette bonne texture }\end{array}$ & $\begin{array}{l}\text { Bons aspects visuels et tactiles } \\
\text { autres que sensations } \\
\text { colorées }\end{array}$ & $\begin{array}{l}\text { Validation de la matière, sa mise } \\
\text { en forme et ses états de surface }\end{array}$ \\
\hline
\end{tabular}

Tableau N 3 - Typologie des maquettes « bonnes sensations »

Cette typologie des maquettes " bonnes sensations " reste compatible et complémentaire de celle de Mer et al. (1995) et Carlile et al. (2004). Mieux, il est utile d'y intégrer les exigences et potentialités du travail collaboratif en mobilisant utilement les travaux de Boujut et Blanco (2002). Non seulement la maquette « bonne sensation » doit se référer aux deux axes définis par Mer et al. (1995) et Carlile et al (2002, 2004), mais nous préconisons de lui devoir lui donner des qualités chronologiquement changeantes en passant d'ouverte à fermée, et de commissionnaire à médiatrice. Pour " fermer » la maquette bonne sensation ouverte, nous avons proposé et validé le fait d'intégrer les connaissances relatives aux techniques d'évaluation de la perception humaine (la métrologie sensorielle en l'orientant design) ${ }^{8}$. La continuité logique de recherche consiste à se rapprocher du processus naturel de

\footnotetext{
${ }^{7}$ Le flou peut provenir de l'attitude du client ou du destinataire, leur prédisposition et leur interprétation. S'il éprouve un enthousiasme spontané, la maquette d'étude change de nature en glissant vers une maquette « bonne pour exécution » (comme le Bon A Tirer de l'imprimerie).

${ }^{8}$ «La recherche en métrologie sensorielle nous permet de conclure à la possibilité de mesurer les aspects perçus d'un produit (tout ou partie), par l'intermédiaire de sujets entraînés (experts), qui, de ce fait, possèdent un jugement reproductible dans le temps. Ils deviennent, alors, un instrument de mesure. Leurs expertises s'exercent dans le cadre d'un espace standard appelé : « cabine d'évaluation sensorielle ». L'évaluation sensorielle aboutit à un profil sensoriel du produit, carte d'identité des sensations du produit, aujourd'hui appelé descriptif sensoriel. Il est constitué d'un espace intermédiaire souvent linguistique
} 
perception et d'imaginer des combinatoires en inter modalités sensorielles, où la maquette visio-tactile médiatrice et ouverte, par exemple, commandera de nouvelles techniques d'évaluation sensorielle à expérimenter et valider.

\section{La maquette bonne sensation, un Objet Intermédiaire à concevoir, réaliser et valider, comme tout autre outil méthodologique du design}

Le projet de conception et de réalisation d'une maquette dans un projet se résume très souvent à une question d'auto réalisation, ce qui peut expliquer l'absence d'explicitation de son cadre de référence. Or, en analogie avec le projet de conception d'un objet, le processus de conception et de réalisation d'une maquette devrait comporter une phase amont de spécifications (de détails d'intention et de résultats espérés), puis une autre liée à la réalisation en elle-même et enfin une dernière phase concernant la validation de la réalisation, c'est-à-dire une comparaison ( une méthode de vérification) qui indiquerait les éventuels écarts existants entre les spécifications ( les performances attendues de la maquette) et les performances réelles constatées ( mesurées, évaluées ) de la maquette réalisée. Cet effort d'explicitation représente la tendance qui doit guider toute recherche dans le cadre d'action recherche en projet de conception design. Ramené à l'O. I. de conception/design, cela peut se représenter comme un projet dans un projet de conception/design, avec un avant ${ }^{9}$ (spécification/conception), un pendant (réalisation/conception) et un après (validation/décision) (Cf. Fig. $\mathrm{N}^{\circ} 1$ ).

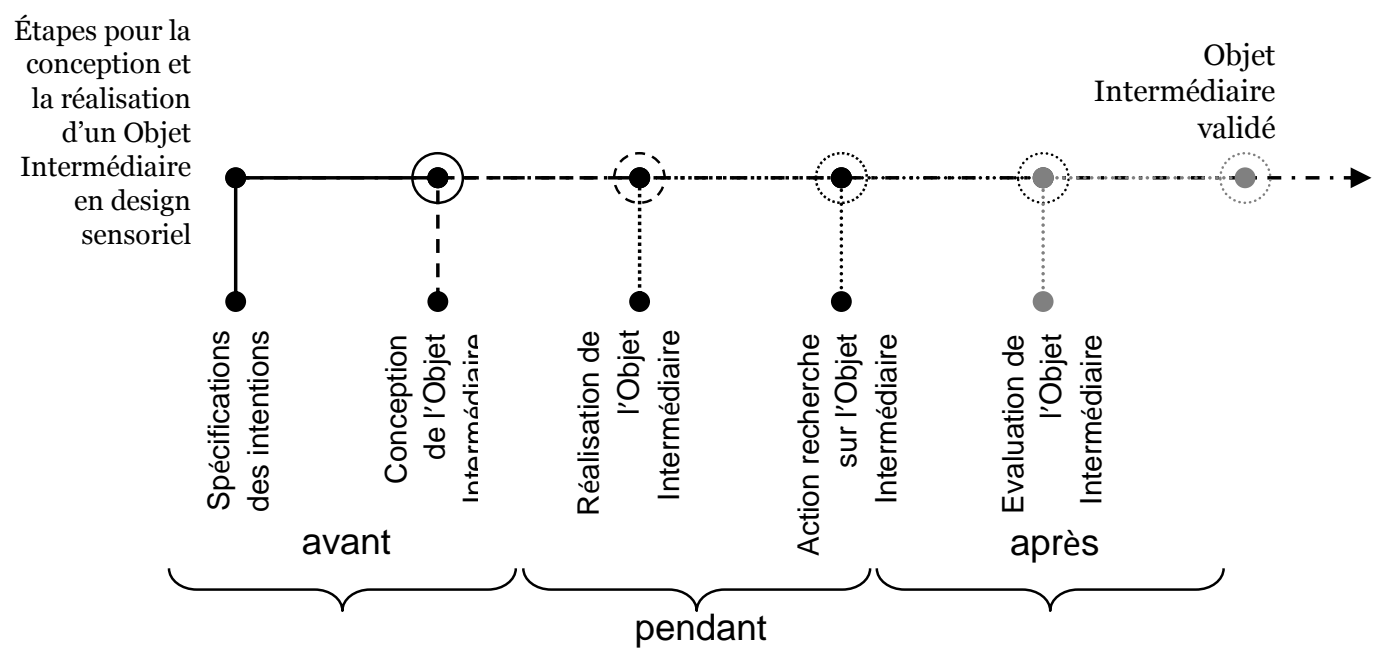

Figure $\mathrm{N}^{\circ} 1$ - Etapes de conception, réalisation et validation d'un Objet Intermédiaire

\section{Les qualités nécessaires d'un 0 . I. de conception/design à concevoir, réaliser et valider}

Comme l'ont défini Mer (1995), Carlile (2002, 2004), Jeantet (1995) et Boujut (1996), l'O. I. oscille sur ce que nous pouvons définir comme deux axes plus ou moins interdépendants ; sur un premier axe, il peut être commissionnaire ou médiateur et sur un deuxième ; ouvert ou fermé. Inscrit en matrice, chaque

composé de descripteurs (qualificatifs ayant subis un protocole de validation (Cf. Normes AFNOR V 09 A et suivantes) auxquels on associe des échelles structurées qui permettent de noter les intensités perçues, en les reliant entre elles. Il est à noter qu'un protocole strict de validation d'une sensation comme grandeur mono dimensionnelle valablement représentée par un descripteur autorise cette mesure originale à l'aide du panel de sujets qualifiés. Différentes épreuves se déroulant dans un espace standard (cabines d'évaluation sensorielle) permettent d'obtenir une réponse représentative du processus de perception humaine ». BASSEREAU. et al. (2003)

${ }^{9}$ Il faut imaginer le résultat final de cette phase avant comme un mode de représentation des performances attendues de la maquette qui fixerait des tolérances et des méthodes de vérification pertinentes comprenant la précision de l'objet de la mesure, les instruments et conditions de mesure. Les modes de représentation spécifiques amorçant le processus de réalisation de la maquette restent présents à ce niveau. Il peut s'agir de dessins, schémas côtés, dans le cas de maquettage conventionnel ou de données numériques permettant le paramétrage de machine de prototypage, maquettage numérique. 
extrême de chaque pôle présente leurs qualités qui ne demandent qu'à être évolutives et combinatoires (Cf. Tableau N4).

$\begin{array}{ccc} & \text { commissionnaire } & \text { médiateur } \\ \text { ouvert } & \mathrm{a} & \mathrm{b} \\ \text { fermé } & \mathrm{c} & \mathrm{d}\end{array}$

Tableau $\mathrm{N}^{\circ} 4$ - Matrice de combinaisons entre pôles des deux axes de définition d'un $\mathrm{OI}$

Plus précisément, la combinaison de ces qualités donne des combinatoires vertueuses et chronologiquement préférables :

a - ouvert/commissionnaire ; lorsque l'Objet Intermédiaire est ouvert et commissionnaire, cela signifie qu'il supporte des modifications tout en étant porteur fidèle de la représentation initiale (qu'elle soit du concepteur ou de sa discipline d'appartenance). Il s'agit souvent de représentation en système, en langage partagée appris ou non (Cf. dessin technique, ou maquette).

La recherche première vise donc à obtenir de tout Objet Intermédiaire des qualités d'ouverture, pré requis à un véritable travail collaboratif. Ce travail peut viser à retrouver en processus la construction de l'objet intermédiaire et à lui donner des existences intermédiaires (Cf. Profil sensoriel analogique idéal)

b - ouvert/médiateur : lorsque l'OI est ouvert et médiateur, il supporte des modifications tout en prenant place entre la représentation de celui qui l'a initié et celle à qui il était destinée. Tout outil méthodologique hybridé avant d'être stabilisé passera par ces phases.

c - fermé/commissionnaire : lorsque l'Ol est à la fois fermé et commissionnaire, il ne supporte aucune modification tout en étant porteur fidèle de la représentation du ou des concepteurs. Ce cas de configuration est à privilégier en fin d'étape de projet, lorsque l'Objet Intermédiaire a subi tous ses protocoles d'évaluation et de validation.

d - fermé/médiateur, ce territoire semble peut pertinent à occuper dans le cas d'un outil méthodologique pour un travail collaboratif ${ }^{10}$.

Nous venons d'expliciter le processus générique d'obtention d'un 0 . I. II suit une métamorphose riche au sein de toute étape d'un projet de conception/design $d^{\prime}{ }^{\prime}$ objet $^{11}$. De commissionnaire disciplinaire souvent fermé, il doit s'ouvrir en médiateur pour se fermer à nouveau mais à la seule condition qu'il soit de nature commissionnaire inter disciplinaire (Cf. Fig. $\mathrm{N}^{\circ} 2$ ). L'intelligibilité de l'hybridation des nouveaux outils méthodologiques interdisciplinaires étant en voie de réalisation, sa faisabilité devient envisageable en suivant le chemin tracé par l'explicitation du processus d'obtention d'un O. I.

\footnotetext{
${ }^{10}$ l'O. I. ne supporterait plus aucune modification tout en se situant dans un entre-deux représentationnel ? Par contre, pour un objet (définitif) cet entre-deux représentationnel peut être souhaitable.

${ }^{11}$ Dans les cas d'hybridation interdisciplinaire, comme dans ceux d'outil métier disciplinaire à rendre intelligible à d'autres acteurs dans un contexte de projet collaboratif, en design particulièrement, le chemin est tracé ; tout outil méthodologique doit être mis en état « $\mathrm{b} »$ (potentiellement modifiable dans un état intermédiaire de représentations multiples possibles), puis en «a », pour faciliter le travail collaboratif, tout en lui garantissant une seule représentation possible partagée, avant d'être validé et figé en « c ». Dans l'objectif d'opérationnalité, l'expérience et la pratique montre la pertinence d'ouvrir et de rendre expansif tout outil méthodologique dans les phases préparatoires aux projets. Les systèmes ouverts favorisent une pensée et une conception transverse, conforme à l'heuristique du projet. Mais cette ouverture ne doit durer que le temps des échanges et des premières marches de la conception préliminaire.
} 


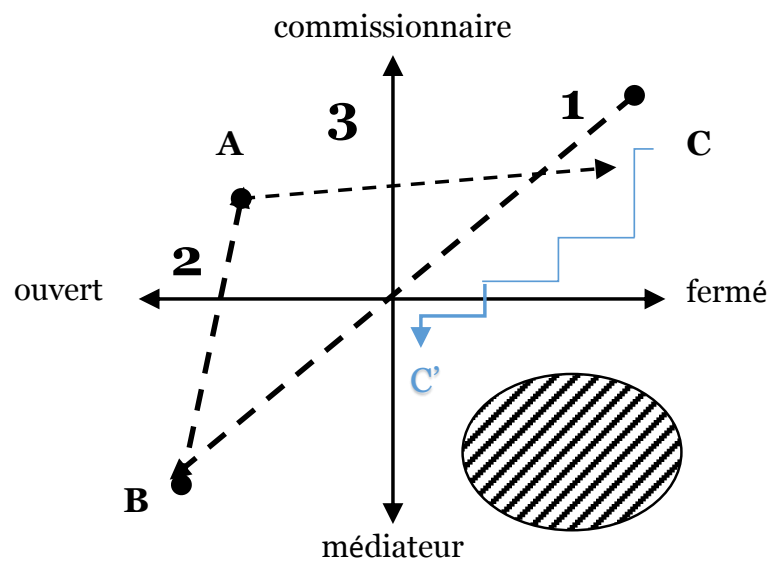

Zone réservée à l'objet final

Figure $\mathrm{N}^{\circ} 2$ - Trajectoire vertueuse d'un Objet Intermédiaire particulier (outil méthodologique) dans le cas de travail collaboratif

L'OI en 1 (commissionnaire/fermé) vers (ouvert/médiateur)

L'OI en 2 (ouvert/médiateur) vers (ouvert/ commissionnaire)

L'OI en $3^{12}$ (ouvert/ commissionnaire) vers (commissionnaire/fermé)

Deux voies d'amélioration concernant l'outil méthodologique sont alors possiblement praticables :

$\left.1^{\circ}\right)$ soit l'outil méthodologique existe et il s'agit de vérifier si il est le véhicule idéal pour mener un travail collaboratif interdisciplinaire, et éventuellement lui apporter les modifications nécessaires pour le rendre plus ouvert et plus médiateur.

$2^{\circ}$ ) Soit l'outil méthodologique n'existe pas. Il est donc à concevoir, ou à hybrider (Cf. Profil sensoriel analogique) en suivant la trajectoire vertueuse de l'Objet Intermédiaire (Cf. Fig. $\mathrm{N}^{\circ} 2$ ), puis le valider dans des contextes de projets de conception/design.

Le processus de métamorphose d'un $\mathrm{O}$. I., pour les outils méthodologiques hybridés, abrite deux grandes tendances dans cette hybridation à concevoir et réaliser :

$\left.1^{\circ}\right)$ soit l'effort est fait pour aller vers l'autre discipline ou domaine qui devient le cadre de référence et $d^{\prime}$ accueil.

$2^{\circ}$ ) Soit l'effort est fait pour attirer l'autre discipline vers le design par la captation de tout ou partie d'outils, méthodes, représentations, voire concepts.

Ce deuxième cas est illustré par le profil sensoriel analogique, issus du profil sensoriel attiré par le design sensoriel en projet de conception/design. Le profil sensoriel reste, à ce jour, l'outil le plus achevé de l'évaluation sensorielle (Cf. Fig. $\mathrm{N}^{\circ} 3 \mathrm{a}, \mathrm{b}, \mathrm{c}$ ). Il permet de disposer d'une cartographie des performances sensorielles d'un produit tel que perçu. Le profil sensoriel associe les différentes intensités des grandeurs sensorielles situées sur une échelle relative à la perception humaine. Chaque descripteur (qualificatif, participe passé ou présent), après validation ( $C f$. encadré), représente valablement une grandeur sensorielle unidimensionnelle.

\footnotetext{
${ }^{12}$ L'OI final en 3 n'est pas à confondre avec l'OI en 1 du début. En 3, sa qualité de connaissance n'est plus disciplinaire mais interdisciplinaire, fermée pour mieux avancer dans le projet de conception/design.
} 


\section{Protocole de validation de descripteurs représentant valablement les grandeurs sensorielles relatives à un espace produit}

La métrologie sensorielle n'a de raison d'être que dans la mesure des qualités organoleptiques des produits. En métrologie générale on ne sait pas mesurer d'autres grandeurs que celles unidimensionnelles. L'évaluation sensorielle respecte cette loi. Elle tente de faire verbaliser des grandeurs qu'il s'agit par la suite d'affiner pour ne retenir que celles ne possédant qu'une seule dimension. Cette verbalisation est déclenchée par rapport aux différences constatées entre les échantillons de l'espace produits (ensemble d'objets rassemblés sur une relation de similitude triple (fonctionnelle (tout objet ou système qui délivre le même service) ou/et morphologique (tout objet ou système qui possède la même forme) ou/et linguistique (tout objet ou système qui s'appelle de la même façon), Depledt (2009)).

Dans un premier temps, tout type de qualification est recueilli (mot, qualificatif, groupe de mots, participe présent et passé). Par la suite, trois tris consécutifs vont être pratiqués. En théorie et dans les écrits méthodologiques, on préconise de situer les échantillons issus de l'espace produits dans la situation à étudier, dans le contexte choisi, c'est-à-dire, en allant aussi recueillir les avis des différentes personnes en relation avec les produits étudiés. Le recueil de termes se termine lorsqu'aucun qualificatif nouveau n'apparaît plus (au bout d'une dizaine de mois en général), la saturation de l'exercice est atteinte.

Le premier tri dit qualitatif vise à éliminer les termes d'une liste possédant en général (150 à 200 termes). Trois critères président au tri qualitatif :

$\left.1^{\circ}\right)$ Le critère de pertinence : ne sont retenus que les termes appartenant aux modalités sensorielles étudiées?

$2^{\circ}$ ) Le critère qualitatif : tous les termes concernant les aspects quantitatifs sont supprimés (l'entraînement de la manipulation conceptuelle d'échelle d'intensité permet d'atteindre une plus grande précision que celle catégorielle des mots tels que " peu », " plus grand ", " moyen » ou encore " beaucoup »).

$\left.3^{\circ}\right)$ le critère hédonique : tous les qualificatifs concernant des jugements de valeurs sont écartés. Les aspects d'aversion et hédoniques se rapportent à un aspect subjectif au sens de "spécifique au sujet ". A priori, cette variable inter individuelle ne peut convenir à une mesure.

Au terme de ce premier tri persiste une centaine de qualificatifs qui représentent potentiellement $100 \%$ de l'information recherchée. L'approche traditionnelle porte un nouvel effort qui vise à réduire à une vingtaine de termes cette liste, en espérant qu'elle contiendrait au moins $80 \%$ de l'information (on utilise un tri quantitatif (en utilisant un premier principe de fréquence de citation), puis enfin, un tri statistique à l'aide des premières quantifications réalisées par le panel sensoriel (Cf. AFNOR, 2001).

Les termes retenus sont définis de manière consensuelle par les membres du panel sensoriel guidé par un panel leader. Dans les cas les plus favorables ils sont illustrés et décrits par un protocole d'exploration et des échantillons de référence (le référentiel sensoriel).

Chaque grandeur sensorielle est évaluée en intensité par les membres du panel sensoriel. Le panel sensoriel constitue un instrument de mesure construit sur le principe d'une vérification de la répétabilité des réponses et de leurs validations comme justes, précises et discriminantes (AFNOR (2001). Le profil sensoriel est le résultat communicable de ces mesures. Il s'appuie sur le principe d'un ensemble d'échelles représentant la plage perceptible des variations de grandeurs sensorielles, représentée valablement par un descripteur ; terme validé par un protocole (Cf. encadré). L'ensemble des échelles d'intensité indiquée au regard des descripteurs sensoriels constitue le profil sensoriel. Ainsi, il est possible de disposer de données fiables qui permettent la comparaison, le positionnement et d'exprimer, en les traduisant, des sensations attendues (profil sensoriel idéal). Plusieurs formalismes coexistent : profil (Cf. Fig. 3 a), histogramme (Cf. Fig. 3 b), diagramme radar (Cf. Fig. 3 c). 

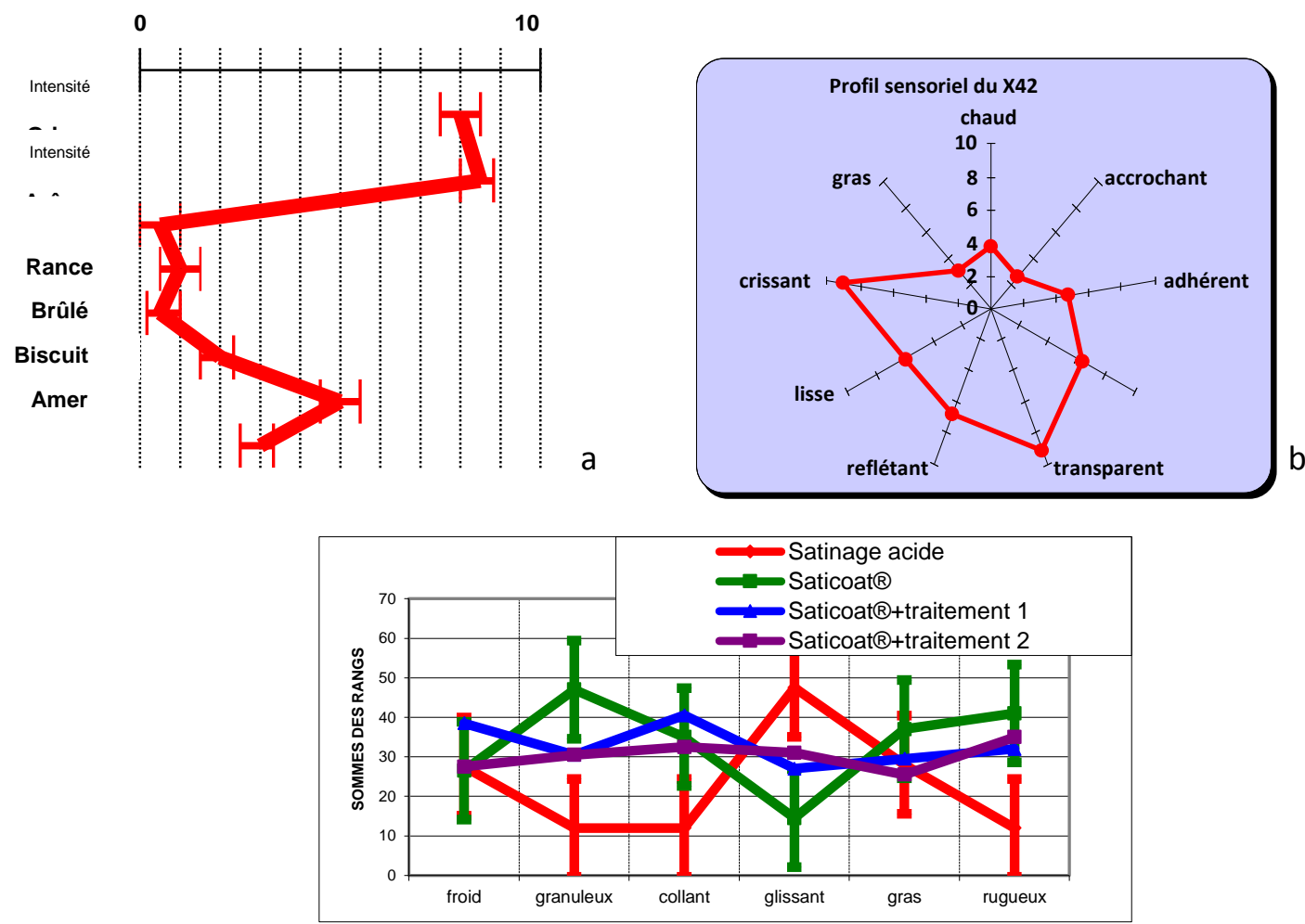

C

Figure $\mathrm{N}^{\circ} 3$-Différents formalismes du profil sensoriel

Pour obtenir un profil sensoriel, il faut avoir initié un effort de description dans un objectif d'évaluation d'une sensation. Cette évaluation sensorielle repose sur quatre grands principes :

$1^{\circ}$ ) le principe de caractérisation des sensations relativement à une famille d'objets, d'où la nécessité de construire un espace " produits " présentant de nombreuses différences entre les entités pourtant réunis dans un lien supérieur d'appartenance catégorielle.

$2^{\circ}$ ) le principe d'évaluation des sensations via un groupe de personnes mobilisant les performances de leur appareil perceptif, d'où l'impérieuse nécessité de supprimer tous les éléments de jugement de valeur et éléments hédoniques spécifiques à chaque personne.

$3^{\circ}$ ) le principe de confier à un mot le rôle de représentation d'une sensation ${ }^{13}$, permet, une fois identifiée cette relation de représentativité (Cf. Grandeur nominale, grandeur ordinale), d'associer dans le cas de grandeurs ordinales, une échelle de variation d'intensité, à " $\mathrm{n}$ » intervalles (Cf. Fig. $N^{\circ} 3 \mathrm{a}, \mathrm{b}, \mathrm{c}$ ).

\footnotetext{
${ }^{13}$ Les caractéristiques identifiantes ramenant à l'évocation d'une matière peuvent très souvent être décrites plus finement encore (Soyeux renvoie à Soie), mais la soie possède des spécificités sensorielles sonores, thermiques, kinesthésiques qui la caractérisent plus finement. Pour les caractéristiques identifiantes (utilisant l'association à une matière de référence), on note deux types d'approches :

$1^{\circ}$ ) l'approche expansive (liée à une texture, par exemple, on peut avoir strié, ondulé, cannelé), où le champ couvert est issu de l'association sémantique des termes (Cf. annexe extrait de l'introduction des mots du sensoriel).

$2^{\circ}$ ) L'approche concentrée (du type cire, bougie, paraffine), où la définition apparaît de l'intersection sémantique (Cf. DACREMONT C. XIème journée d'analyse sensorielle, «La référence sensorielle », Cergy Auditorium Espace St Louis, 10 juin 2008).
} 
$4^{\circ}$ ) le principe de mesure dans un contexte maîtrisé, où les variables exogènes à l'objet de mesure sont neutres et sans influence. La cabine d'évaluation sensorielle vise à atteindre ces conditions de mesure.

L'ensemble permet de réaliser une caractérisation sensorielle d'un objet ou échantillon de produit, de produit semi fini, de matière. Mais ces pratiques dans leurs logiques d'évolution observées questionnent quant à l'effort de métrologie, son positionnement, et son caractère par rapport au déroulement du projet de conception/design d'un objet. Trop de précision ne nuirait-il pas en conception préliminaire, étouffant toute créativité ?

\section{Le profil sensoriel analogique illustré par la maquette sensorielle, un exemple d'O. I. de conception issu d'une recherche par le design sensoriel}

Le profil sensoriel analogique est né face à la nécessité de disposer très tôt dans le projet de conception/design de spécifications sur les performances attendues des propriétés organoleptiques de l'objet à concevoir, spécifications à situer au même niveau d'information que celles du Cahier des charges des concepteurs plus techno centrés. Dans sa construction, le profil sensoriel analogique a le mérite de se rapprocher d'un fonctionnement plus naturel de la perception (relativité et comparaison). Il est par nature évolutif. Une fois déterminé le premier niveau de la grandeur ordinale, représenté par un ensemble d'intervalles et de seuils (éventuellement illustré (pré maquette sensorielle)), il est possible de l'affiner en niveau plus élevé (augmentation des intervalles) ${ }^{14}$.

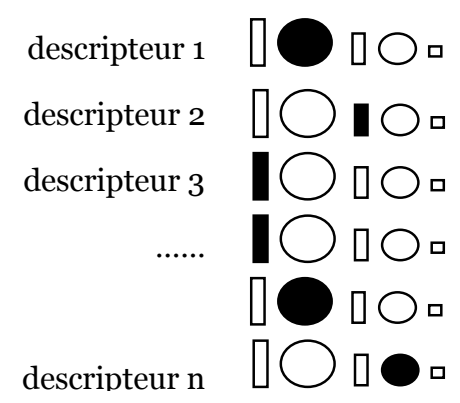

Figure $\mathrm{N}^{\circ} 4$ - Profil sensoriel analogique Niveau 1

Le profil sensoriel analogique permet d'exprimer des intensités (ou plage d') souhaitées en fixant un cadre à la conception ; le designer sensoriel peut choisir de " fermer " certaines plages d'intensité, en les interdisant (codifiées en rouge) ou les souhaitant fortement (codifiées en bleu), pour contraindre le choix de matériaux qu'ils ne maîtriserait pas, ou de laisser " ouverte " telle ou telle plage d'intensité (codifiées en blanc). De nombreux métiers président à l'obtention d'effets perçus. Ils disposent, grâce au profil sensoriel analogique idéal, d'un $\mathrm{O}$. I. de conception, qui spécifie, favorise le dialogue interdisciplinaire, arbitre, et statue sur la question des propriétés organoleptiques de l'objet à concevoir.

\footnotetext{
${ }^{14} \mathrm{D}$ 'autant plus si les échantillons à évaluer sont nombreux et présentent des différences dans leurs propriétés organoleptiques et/ou que le panel sensoriel augmente ses performances discriminative Les praticiens de l'évaluation sensorielle ont maintes fois constaté l'incroyable possibilité d'augmentation de la performance humaine par l'apprentissage et l'entraînement. Du fait qu'une des fonctions naturelles du processus de perception humaine concerne son aspect comparatif et relatif. Ainsi, très naturellement, les intervalles vont être représentés en taille plus réduites mais en nombre plus grand dès qu'une catégorie d'échantillons présentera des différences d'intensité de la grandeur sensorielle représentée.
} 
II n'y a pas de limites de division d'une échelle d'intensité, tant qu'elles n'ont pas été identifiées, représentées, illustrées par au moins un échantillon. Dès qu'il y a modification du nombre d'intervalles, celle-ci est signalée par le changement de dénomination d'un niveau (Cf. Fig. $\left.\mathrm{N}^{\circ} 5\right)^{15}$.

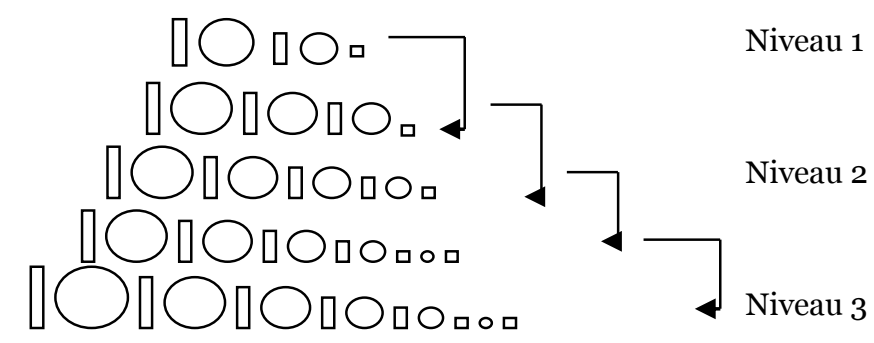

Figure $\mathrm{N}^{\circ} 5$ - Illustration d'un apprentissage progressif réussi d'un panel sensoriel capable de subdiviser des intervalles jusqu'à un niveau de précision de 5 à 6 intervalles (7 seuils)

Le passage d'un niveau amont à un niveau aval ne peut se réaliser que si l'existence d'un consensus entre tous les sujets du panel est identifiée, à moins qu'une variable biologique explique cette impossibilité ${ }^{16}$. "L'étude progressive permet de mener une démarche dynamique intégrant les résultats intermédiaires " Lattuf (2006). II faut souligner que le profil sensoriel s'assimile à une photographie, une représentation dans un temps arrêté. Le plus souvent à une ou deux modalités sensorielles additionnées. ${ }^{17}$ II reste un champ important à explorer qui illustrerait une vraie représentation sensorielle intermodale.

\section{Conclusion sur les objets Intermédiaires co construits comme résultat attendus des outils méthodologiques existants et à concevoir du design sensoriel}

L'Ol est non seulement le véhicule idéal pour initier et prolonger un dialogue interdisciplinaire dans les projets de conception/design, mais ceux issus du design, par essence, s'y prêtent d'autant mieux qu'ils $s^{\prime}$ adressent quasi naturellement à la perception humaine. Les recensements renseignés des Ol que nous avons initiés en les décrivant dans le prolongement du travail de Mer, notamment sur l'explicitation de leurs cadres d'appartenance, ouvre la voie à une prolongation fructueuse. Parmi l'ensemble des (re)présentations intermédiaires existantes, d'autres recherches futures sont à initier, sur les outils méthodologiques notamment, en atteignant les qualités que nous avons explicitées d'un 0 . I. de conception (Cf. Fig. $\mathrm{N}^{\circ} 2$ ), illustré par le profil sensoriel analogique. Mais sur ce chemin, en Action Recherche, tout OI n'est pas toujours connecté à un outil méthodologique. Toujours est-il que tout outil

\footnotetext{
${ }^{15}$ En changeant de niveau, tous les seuils intermédiaires peuvent (ou non) changer ; seuls les seuils mini et maxi, s'ils ont été représentés dans un absolu, restent inchangés. Excepté, bien entendu lorsque l'espace produit reste relatif dans ses propriétés organoleptiques. Mieux, les niveaux peuvent se représenter schématiquement pour indiquer éventuellement les absences d'échantillons qui auraient pu rendre complet l'illustration d'une variation des intensités d'une grandeur sensorielle ordinale (Cf. Fig. $\mathrm{N}^{\circ} 5$ ).

${ }^{16}$ Cette possibilité n'est pas la moindre des qualités évolutionnistes du profil sensoriel analogique. Pouvoir identifier la rupture de consensus dans une réponse, un classement ordinale d'une grandeur sensorielle renseigne à différents niveaux : à un niveau individuel, pour le panéliste débutant se peut être le signal d'un manque de discrimination. A un niveau panel sensoriel, se peut être le début d'une recherche d'explication et de prise de conscience de la relativité d'une mesure en perception, de variables endogènes à la personne (biologique, physio-sociologique) qui reposeraient la problématique de la représentativité d'une population. Par exemple, la sudation est variable suivant les contextes et les personnes, une représentation en courbe de gausse de la population renseignerait sur des exigences de représentation des percentiles de celles-ci dans un panel sensoriel.

${ }^{17}$ Même si le profil Spectrum vise à une exhaustivité quant à la représentation des modalités sensorielles et leurs grandeurs associées, Cf. BARTHELEMY J., DANZART M., MAC LEOD P., SAUVAGEOT F., - «Evaluation sensorielle Manuel méthodologique ", éd. Technique et documentation de Lavoisier, 1 ère édition 1990, $2^{\text {ème }}$ réédition 1998. Il est intéressant que le profil sensoriel Spectrum ne soit plus mentionné dans les guides de bonnes pratiques, ni même la réédition du manuel méthodologique d'évaluation sensorielle, car trop coûteux en temps.
} 
méthodologique ayant des prétentions d'opérationnalité en projet collaboratif de conception/design $d^{\prime}$ objet se doit de respecter, non seulement le critère de médiation mais tous les critères d'un 0 . I. que nous avons explicité, et notamment s'adapter pour respecter le processus de partage de tout outil de représentation intermédiaire interdisciplinaire ; caractère ouvert ou fermé de l'O. I. gage d'un partage interdisciplinaire (le pré requis de la co construction), par la prise de conscience de la relativité de l'effort de représentation et représentativité de tout ou partie de l'objet, son contexte et ses interactions avec leurs utilisateurs), et en rendant expansif en transdisciplinaire les variations qui en émanent ${ }^{18}$. Quelles qu'elles soient, les qualités des 0 . I., définies dans le cadre de référence théorique de l'action recherche, peuvent être réduites au nombre de trois (Cf. Tableau $\mathrm{N}^{\circ} 5$ ) :

\begin{tabular}{|l|l|}
\hline A & ouvert/commissionnaire $^{19}$, \\
\hline B & ${\text { ouvert } / \text { médiateur }^{20},}$ fermire $^{21}$, \\
\hline C & fermé $^{1}$ commissionnais \\
\hline
\end{tabular}

\section{Tableau $N^{\circ} 5$ - Processus vertueux A-B-C des qualités augmentées d'un Objet Intermédiaire}

Dans ce contexte d'action recherche par le design, une logique d'évolution s'instaure. Ainsi, si L'Ol est disciplinaire, il est souvent (commissionnaire/fermé). Un programme de recherche orientée en, pour et par le Design (Findeli, 2008) viserait tout en l'hybridant à expliciter ses états de construction successive (et donc le faire exister en état de pré maturation), pour le rendre à la fois ouvert (acceptant des variations) et médiateur entre différentes disciplines. Au cours de l'avancée du projet, l'OI va évoluer pour toujours accepter des modifications de moins en moins fréquentes et importantes et devenir commissionnaire non plus d'un point de vue disciplinaire mais interdisciplinaire (Cf. Fig. $\mathrm{N}^{\circ} 2$, noté $\mathrm{C}^{\prime}$ ). Ainsi, chaque outil méthodologique suit une progression représentationnelle traçable, respectant les mutations de tout $\mathrm{O}$. I., évoluant au sein du projet. Une des voies d'une recherche par le design viserait à appliquer ce processus mutationnel et évolutif à toute représentation intermédiaire ${ }^{22}$.

${ }^{18}$ La page n'est pas blanche. De nombreux outils méthodologiques ont déjà été expérimentés en projets collaboratifs (CERTESENS, Bassereau et al., 1995 à 2015). Soient, ils ont été attirés vers le design sensoriel par transfert, modification, transformation (profil sensoriel analogique, référentiel sensoriel), soient ils ont été conçus, testés et modifiés jusqu'à leurs validations opérationnelles (Cf. arbre généalogique, par exemple, Bassereau, 1995 et 2001). In fine, tous ces outils méthodologiques aboutissent à une ou plusieurs représentations intermédiaires issues ou non d'une hybridation transdisciplinaire. L'expérimentation d'une nouvelle hybridation d'outil méthodologique doit respecter les qualités de tout outil méthodologique qui s'inscrit dans un projet interdisciplinaire de conception design et posséder au moins un Objet Intermédiaire qui satisfasse les qualités de ceux- ci. Rappelons les qualités que doit posséder un outil méthodologique collaboratif :

$\left.1^{\circ}\right)$ s'inscrire de manière efficiente dans toutes les étapes du projet et permettre son évolution et son évaluation, y compris son résultat final ; l'objet (action),

$2^{\circ}$ ) faciliter la co construction interdisciplinaire dans le cadre d'un projet collaboratif, puis le dialogue

interdisciplinaire, voire la génération de questions de recherche à objet de recherche trans, inter ou uni disciplinaire, $3^{\circ}$ ) provoquer la génération d'idées et son processus de travail créatif et de conception,

$4^{\circ}$ ) augmenter la connaissance immédiate et située (action), en facilitant une connaissance construite plus générique (recherche).

${ }^{19}$ Lorsque l'O. I. est ouvert et commissionnaire, cela signifie qu'il supporte des modifications tout en étant porteur fidèle de la représentation initiale (qu'elle soit du concepteur ou de sa discipline d'appartenance). Il s'agit souvent de représentation en système, en langage partagée (Cf. dessin technique, ou maquette).

${ }^{20}$ Lorsque l'OI est ouvert et médiateur, il supporte des modifications tout en prenant place entre la représentation de celui qui l'a initié et celle à qui il était destinée.

${ }^{21}$ Lorsque l'OI est à la fois fermé et commissionnaire, il ne supporte plus aucune modification tout en étant porteur fidèle de la représentation du ou des concepteurs.

${ }^{22}$ Il existe des (re)présentations intermédiaires qui résistent à devenir des outils méthodologiques, mais l'inverse ne peut être vraie Certains outils méthodologiques restent disciplinaires par manque de maturité, mais d'autres sont en butte à des problèmes d'explicitation liés à une incorporation trop profonde des savoirs et des connaissances. Ce sont souvent des connaissances où l'appareil perceptif humain guide la « main » qui devient pensante, dans la transformation, l'assemblage de matériau, par exemple. Il existe aussi des cas où l'outil méthodologique colle à sa représentation et dans cette indissociabilité entraîne l'acteur chercheur dans une pratique dogmatique de la discipline. C'est par exemple le cas de l'utilisation des descripteurs (grandeurs ordinales) en évaluation sensorielle descriptive. Or, dans des logiques projets tout gain de temps est souhaitable s'il ne fait pas perdre de l'information, de la confiance et des données. La génération de descripteurs représentant valablement les sensations à évaluer consomme du temps. Pour les modalités sensorielles tactiles, visuelles et sonores une catégorie récurrente de descripteurs a été établie, Bassereau et Charvet Pello (2011). Nous les avons appelés des grandeurs 


\section{Bibliographie}

AFNOR, (2001) « Analyse sensorielle - 6 ème édition ", AFNOR, novembre BASARAB N., (1996) - "La transdisciplinarité », manifeste, Éd. du Rocher BASSEREAU J.F., CHARVET PELLO R. (2011) - « Dictionnaire des mots du sensoriel », éd. Lavoisier, Paris, avril

BASSEREAU J.F., CHARVET PELLO R., BONNAMY L. (2009) - « Le design sensoriel », éd. Techniques de I'Ingénieur, Paris

BASSEREAU JF., LATUFF J. A., DUBOIS P., DUCHAMP R. (2003) - « Maquette « Bonne Sensation » (M.B.S) ", Colloque CPI 2003 à Mekhnès (Maroc)

BAUDIN C. (2003) - "Produits conçus, objets vécus -une approche ethnométhodologique sensible des pratiques de conception et d'usage ", Thèse de doctorat, Université de Paris 7

BOUDON P, POUSSIN F. (1988) - " Figures de la conception architecturale », éd. Dunod, Paris

BOUJUT J.F., JEANTET A. (1998) - " Les entités de la coopération dans les nouvelles organisations de la conception ", Performance Humaines et Techniques, $\mathrm{N}^{\circ} 96, \mathrm{pp} 38-44$

BOUJUT J.F., CAVAILLE J.B., JEANTET A. (2000) - «Instrumentation de la coopération », Prospers, Journées de Toulouse, 7-8 juin, pp 102-110

BOUJUT J.F., BLANCO E., - « Intermediary objects as a means to foster cooperation ", in Actes de la Conférence : Engineering design, Journal of computer supported collaborative work, Vol. 12 Issue 2, PP 205- 219

CARLILE P. R. (2002), - « A pragmatic view of knowledge and boundaries: Boundary objects in new product development ", Organization science, 13(4), 442-455

CARLILE P. R. (2004), - " Transferring, translating and transforming knowledge: An integrative framwork for managing knowledge across boundaries ». Organization Science, 15(5), 555-568. CARAES M. H., MARCHAND ZANARTU N. (2011), - «Images de pensée ", éd. RMN, Paris

CIHUELO J. (2002) - « Projet de conception et processus Coopératif : Les « petits Riens » qui rassemblent les métiers ", Revue annuelle des Arts et Métiers, Paris

COSTES E., (2012) - « Prise en compte de la perception tactile dans la conception de représentations d'oeuvres d'art pour les personnes en situation de handicap visuel ", thèse de doctorat ENSAM, Paris, DEFORGES Y., (1981) - "Le graphisme technique son histoire et son enseignement ", éd. Champ Villon DEPLEDT F. (2009) - « Manuel méthodologique d'évaluation sensorielle », $3^{\text {ème }}$ édition, Ed. Lavoisier, juin, Paris

DUBUISSON S., HENNION A. (1998) - « Le design : l'objet dans l'usage; la relation objet-usage-usager dans le travail de trois agences ", Ed. Les Presses de l'Ecole des Mines de Paris, FINDELI A. (1998) - " la recherche en design : questions épistémologiques et méthodologiques", International Journal Of Design and Innovation, vol. $1 \mathrm{~N}^{\circ} 1$, juin FINDELI A. (2008), Introduction aux Ateliers de la Recherche en Design, Nîmes, juin

FINDELI A. (2008) - "Transdisciplinarity; a tentative contribution to the methodology of design research ", Swiss Design Network Symposium 2008, Berne, 30-31 mars

FILLACIER J. (1986) - « La pratique de la couleur », éd. Dunod, Paris

HATCHUEL A. (2006) - "Quelle analytique de la conception ? Parure et pointe en design ", Le design essais sur des théories et des pratiques, direction FLAMAND B., IFM, éd. du regard, Paris JEANTET A. (1998) - « Les objets intermédiaires dans la conception. Eléments pour une sociologie des processus de conception ", Sociologie du Travail, 3-98, pp 291-316

sensorielles génériques pour les différencier de celles spécifiques (à l'objet, au contexte). L'existence de ces grandeurs permet de faire appel à des pré listes établies. L'effort sur la génération de descripteurs spécifiques se produit très naturellement, lorsque, malgré les caractérisations en grandeurs génériques, deux produits perçus comme différents restent classés à l'identique. Seules alors, de nouvelles dimensions sensorielles pourront décrire ces différences. Cette génération de termes avec support permet aussi de faire prendre conscience plus aisément à tous que la réalité est différentes des efforts de taxonomie de l'évaluation sensorielle. 
LATTUF J.A. (2006), - « Aide au pilotage d'une démarche d'innovation en conception de produits : vers un cahier des charges "augmenté» ", thèse de doctorat ENSAM, Paris, 18 décembre MAGNON L. (1991) - " Méthodes du design pour la création industrielle ", Design Recherche Avril MER S. JEANTET A. TICHKIEWITCH S. (1995) - « Les objets intermédiaires de la conception :

modélisation et communication ", Le communicationnel pour concevoir, Europia , Ed. Caelen Jean et Zreik Khaldoun.

MINEL S. (2003) - " Démarche de conception collaborative et propositions d'outils de transfert de données métier ", Thèse de doctorat ENSAM, Paris

MORIN E. (1999) - "Relier les connaissances, le défi du XXIème siècle ", éd. du Seuil, Par

PESCE G. (1996) - " Le temps des questions ", éd. du Centre G. Pompidou, Paris, ISBN 2-85850-884-4

PROST R. (sous la direction de) (1995) - «Concevoir, inventer, créer », éd. L'Harmattan, Paris, ISBN 27384-3610-2.

QUARANTE D. (1995) - "Eléments de design industriel », éd. Maloine $1^{\text {ère }}$ éd. (1984), $2^{\text {ème }}$ ré éd. RIVERE , BASSEREAU J. F., BERTHELOT S., WANET S., PEYERCE J., HARRIS N. (2008) - « Emballages de liquides alimentaires : la conception de la recommandation comme outil de transfert spécifique des résultats d'une recherche vers le processus de création ", Société en Ergonomie de Langue Française, SELF, Ajaccio, septembre

SCHWACH V. (1994) - "Les indicateurs micropsychologiques de la satisfaction de l'utilisateur", Colloque sur la perception de la qualité ; méthodes et outils d'évaluation de la qualité perçue MESR 13 Juin, Paris

SIMON N. (2006) - "Une agence de design : Construction de croyances et de pratiques autour de l'observation et la création " thèse de doctorat, Université de Paris 7

STOELTZLEN N. (2004) - " Intégration de la dimension visiographique dans les phases amont du processus de conception de produits mécaniques pour favoriser la coopération et la construction commune de projet ", Thèse de doctorat ENSAM, Paris

TICHKIEWITCH S., JEANTET A., MER S. (1995)- « Les objets intermédiaires de la conception : modélisation et communication ", in Le communicationnel pour concevoir, pp 21-41, Europia, Ed. Caelen Jean et Zreik Khaldoun

VINCK D., JEANTET A., - "Mediating and Commissioning objects in the Sociotechnical Process of Product Design : a Conceptual approach", in D. VINCK et al., Management and New Technology Design networks, Strategy, COST Social Sciences serie, CCE, pp 111-129 